\title{
EDITORIAL
}

\section{HACIA NUEVOS MARCOS DE REFLEXIÓN. V CONGRESO NACIONAL DE HISTORIA DE LA ENFERMERÍA}

(SEVILLA, 25-27 DE OCTUBRE DE 2001)

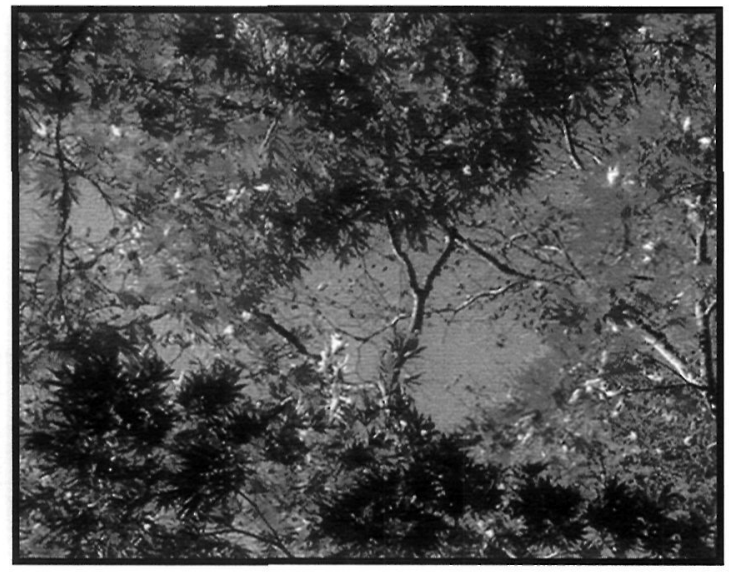

¿Puede constituir el estudio de la Historia de la Enfermería un espacio de reflexión válido para el desarrollo de diversos aspectos que ayuden a situar a la profesión en el nivel al que aspiran sus profesionales? ¿Hasta qué púnto el conocimiento y difusión entre los propios enfermeros y entre el resto de la población de todo aquello que los profesionales de la Enfermería han realizado a lo largo de los siglos por mejorar la calidad de vida de los ciudadanos puede contribuir a que este colectivo mejore su imagen tanto dentro como fuera del grupo profesional?

En este contexto debe situarse la investigación sobre la Historia de la Enfermería desarrollada en los últimos años por distintos grupos de enfermeros y especialistas de otras disciplinas, que están aportando interesantes y abundantes materiales para la reconstrucción de la Historia de la profesión. Aquí habría que situar la celebración durante los días 25, 26 y 27 de octubre en el hotel A1Andalus Palace de Sevilla del V Congreso
Nacional de Historia de la Enfermería. Cinco congresos promovidos desde el Seminario Permanente para la Investigación de la Historia de la Enfermería (adscrito a la Universidad Complutense de Madrid) en los que una disciplina joven está acaparando día a día el interés de profesionales de la Enfermería e investigadores de otras disciplinas científicas: Historia, Antropología y Medicina, especialmente, que están consiguiendo, en una colaboración interdisciplinar, importantes avances para el conocimiento del rico pasado de la profesión.

En este V Congreso, más de dos centenares de investigadores de la mayor parte de las Comunidades Autónomas españolas, además de Brasil y Chile, han presentado los avances de sus investigaciones, entrando en contacto distintos grupos de trabajo, ya consolidados muchos de ellos, en fase de consolidación, otros, de toda la geografía nacional, descubriéndonos, a su vez, las posibilidades de la investigación histórica como elemento aglutinador de los profesionales de la Enfermería, consolidación de la identidad profesional y revalorización de una profesión en el seno de la sociedad en la que desarrolla su actividad diaria.

\section{DESARROLLO DEL CONGRESO}

Bajo el lema "Tradición y Modernidad: las fuentes para el estudio de la Historia de la Enfermería", se propusieron a los investigadores interesados en participar en el Congreso cinco apartados temáticos, a partir de los cuales aportar la mayor cantidad y diversidad posible de fuentes históricas que pudiesen servir de base para posteriores investigaciones en la materia. Así, el Congreso quedó estructurado en los siguientes apartados: 
1) Textos didácticos y docentes.

2) Textos normativos.

3) Textos administrativos.

4) Textos narrativos.

5) Otras fuentes para la Historia de la Enfermería.

La propuesta ofrecida desde la Organización del Congreso obtuvo un eco muy favorable. El Comité Científico ha aceptado 109 comunicaciones (91 orales y 18 posters), que, junto a la Conferencia Inaugural (impartida por la profesora de Paleografía y Diplomática de la Universidad de Sevilla D.ña María Luisa Pardo Rodríguez), las cuatro Ponencias (impartidas por los profesores D. Francisco Herrera Rodríguez, de la Universidad de Cádiz, D. José Siles González, de la Universidad de Alicante, D. Cecilio Eseverri Chaverri, de la Orden Hospitalaria de San Juan de Dios, y D.ña Magdalena Santo Tomás Pérez, de la Universidad de Valladolid), una Mesa Redoda (con la participación de D.ũa Francisca Hernández Martín, de la Universidad Complutense de Madrid, D. Francisco Ventosa Esquinaldo, de la Orden Hospitalaria de San Juan de Dios, y D.ña Amparo Nogales Espert, de la Universidad Rey Juan Carlos), y la presentación de las Actas del IV Congreso Nacional de Historia de la Enfermería, celebrado en Gijón en 1999 (número monográfico de Híades. Revista de Historia de la Enfermería) y el número 1 de la Biblioteca de Clásicos de la Enfermería Española (nueva colección de textos para el estudio de la profesión enfermera), constituyen el eje científico de esta cita en Sevilla con la Historia de la Enfermería.

Ha quedado puesto de manifiesto a lo largo de las distintas sesiones celebradas la amplia diversidad de líneas de investigación emprendidas y desarrolladas en la actualidad por investigadores y grupos de investigación, contribuyendo ello a un mayor y mejor conocimiento del pasado de la profesión enfermera. Se han abordado temas como la complejidad de la reconstrucción histórica en base a los textos escritos; la importancia de un estudio y tratamiento serio y riguroso de las fuentes iconográficas; las aportaciones a la Historia de la Enfermería desde una dimensión multicultural (Enfermería Transcultural) o el análisis de fuentes de la Enfermería en Hispanoamérica.
Enriquecedores resultan, asimismo, trabajos cuya temática se centra en, por ejemplo, la indumentaria de la enfermera a lo largo de la Historia; el estudio de objetos e instrumental empleado por el enfermero en las distintas épocas; textos y manuales docentes para la enseñanza de la Enfermería; estudio sobre la relación arte-enfermería o, por citar algún ejemplo más, la presencia de la enfermería en la filatelia española, desde sus comienzos al presente.

Es interesante destacar que cada comunicación viene elaborada por una media de dos investigadores, que nos hablan en torno a los 250 investigadores de toda España los que han estado presentes (directa o indirectamente en el Congreso), además de los asistentes, entre los que se encontraban 100 estudiantes de las distintas Escuelas de Enfermería sevillanas, becados por la Organización.

En suma, el Congreso ha puesto sobre la mesa el creciente interés que están despertando los avances de la investigación histórica sobre la Enfermería Española, en el seno de la reflexión global que se está produciendo sobre la necesidad de una formación humanística en una sociedad en donde la excesiva tecnificación está generando profundos conflictos emocionales. La presencia de profesionales de toda la geografía nacional y de distintos ámbitos científicos confirman el buen pulso que mantiene la Historia de la Enfermería Española, que tendrá dentro de dos años en Alcalá de Henares su próxima cita. Buena suerte a los organizadores.

Manuel Jesús Garcia Martínez Antonio Claret Garcia Martinez

Presidentes 